\title{
Fontaines narratives de Jean Giono
}

\section{Leforestier, Claire}

Cours Municipaux d’Adultes, Ville de Paris, leforestier.claire@neuf.fr

\begin{abstract}
Resumen
El agua, en la obra de Jean Giono, aunque menos descrita que el aire y la luz, elementos también vitales de los cuales el narrador se empeña en restituir el fascinante poder de animación, presenta múltiples aspectos y cumple funciones esenciales. A través de una sencilla escena de niños, Giono revela, en « Juegos o la naumaquia », como del lecho del agua viva nace la ficción, y como del contacto con el agua nacen al mismo tiempo las criaturas y el creador. Fuente, riachuelo, lluvia, rio, mar... todas las aguas, cada una con su personalidad, están en relación con otros seres o metamorfosis, y su presencia provoca un flujo de eventos, da lugar a una corriente, o deriva, como la narración misma. Más allá de su propiedad genética, el agua "gionesca » se distingue por su naturaleza intermitente, y por tres cualidades frecuentemente asociadas; ruidosa, brillosa y fragante, la materia acuática aparece como una sustancia vibrante, cualidad esencial de la vida, que se relaciona con la personalidad sensible del artista.
\end{abstract}

Palabras clave : agua ; Giono ; creación ; acuático ; narración.

\section{Résumé}

L'eau, dans l'œuvre de Jean Giono, quoique moins "décrite » que l'air et la lumière, autres éléments vitaux majeurs dont le conteur s'emploie à restituer le fascinant pouvoir d'animation, revêt de multiples aspects et assure des fonctions cruciales. Avec une simple scène d'enfants, Giono révèle, dans « Jeux ou la naumachie », que le lit d'eau vive fait celui de la fiction. Du contact avec l'eau naissent en même temps les créatures et le créateur. Fontaine, ruisseau, pluie, fleuve, mer... les diverses eaux, dotées d'une personnalité, sont corrélées à d'autres êtres ou à des métamorphoses. Leur présence amorce un cours d'événements, donne lieu à l'écoulement ou à la dérive : la narration même. Outre sa propriété génétique, l'eau gionienne se singularise par sa nature intermittente, ainsi que par trois qualités couramment associées ; bruyante, chatoyante et odorante, la matière aquatique apparaît comme une substance vibrante. Cette qualité primordiale de l'être apparente l'élément à la personnalité sensible de l'artiste.

Mots-clés : eau ; Giono ; création ; aquatique ; narration.

\begin{abstract}
In Jean Giono's works, water assumes multiple aspects and fulfils crucial functions, although it is less described than air and light, the other major vital elements of which the narrator applies himself to rendering the fascinating ability to enliven things. Through a simple children scene, in «Jeux ou la naumachie », Giono reveals that the banks of flowing waters make the bed of fiction as well. From the contact with water spring at the same time the creatures and the creator. Fountain, stream, rain, river, sea... the various forms of water, endowed with personalities, correlate with other beings or metamorphoses. Their presence initiates a course of events, gives rise to flow or drift: the narration itself. Besides its genetic property, the gionien water distinguishes itself by its intermittent nature, as well as by three qualities generally associated; loud, sparkling and fragrant, aquatic matter reveals itself as a vibrating substance. This essential quality of being relates the element to the artist's sensitive personality.
\end{abstract}

Keywords : water ; Giono ; creation ; aquatic ; narration. 


\title{
Introduction
}

Le chercheur d'eau, en parcourant l'œuvre de Jean Giono, ne manquera pas d'en trouver sous toutes ses formes et dans tous ses états. Son biographe Pierre Citron cite le premier écrit connu de l'adolescent, un sonnet de 1911 qui évoque une " vasque de pierre aux cristallines eaux » et se clôt sur une chute d'eau inspirante : « Je bois la rêverie ainsi que la fraîcheur, / Dans les vagues d'iris des blanches cascatelles » $(1990: 57)^{1}$. Si la voix proprement gionienne n'est pas encore identifiable dans des vers qui font plutôt penser à Jean Moréas² , dès les débuts, l'eau est à la fois sujet d'écriture et matière à images. Elle le restera : Giono écrira sur le fleuve un roman, Le Chant du monde, un film, L'Eau vive, il improvisera sur la mer un récit fantastique: Fragment d'un paradis ; et les images aquatiques ${ }^{3}$ seront parties intégrantes de sa prose. Les observations que je présenterai valent surtout pour les titres précités, les textes de Colline, Jean le Bleu, Que ma joie demeure, Noé, certaines chroniques et nouvelles. Je me propose de mettre en évidence quelques aspects originaux de la présence de l'eau ${ }^{4}$ dans l'écriture de Giono. Deux ouvrages thématiques existent: la thèse d'Ibtissem Bouslama $L a$ poétique de l'eau dans l'œuvre de Giono (1984) et l'étude de Michèle Belghmi Giono et la mer (1987). Bien que rapprocher René Char et Jean Giono n’aille pas de soi, j’ai emprunté au poète de la Sorgue sa belle formule « fontaine narrative » pour intituler ma communication, tant l'image convient à la loquacité intarissable du romancier, et aussi parce qu'elle associe l'eau à la genèse du récit, sujet dont je parlerai d’abord, avant de considérer les eaux diverses puis la matière aquatique.

\section{L’eau génétique}

La propriété génétique de l'eau, universellement reconnue, se manifeste sous bien des aspects. L'eau est une matrice naturelle de formes et de vie: elle érode, irrigue, c'est un bouillon germinatif. Giono représente ces actions. Mais le temps manquant pour traiter l'ensemble des aspects génésiques, je parlerai seulement du plus personnel d'entre eux, du plus " gionien », que l’on pourrait appeler « poïétique », qui réside en cela : le contact avec une eau vive éveille l’imaginaire et amorce le récit. L'écrivain le révèle d'abord dans « Jeux ou la naumachie », récit publié en 19225. C’est une simple scène d'enfants au bord de la rivière. Le narrateur se remémore un phénomène subit de projection : « [...] en moi se dresse une image qui soudain m’illumine : la grande mer !» (1999 : 64). Sous l'effet de cette image-écran, il façonne et met à flot un bateau de roseau, puis toute une flottille :

\begin{abstract}
Si le bois empenné est le bateau, je serai, moi, le dieu qui préside [...]. Dans le coin d’ombre où je suis assis, les berges sont rectilignes comme les quais d'un port civilisé. L'étoffe verte et bleue moirée de l'eau se déroule et, dansant dans le creux des vagues minuscules, au large, une sargasse de feuilles mortes s'étale. [...] Au milieu du désert nu de l'eau, la chevelure du fond monte en ondulant étaler au soleil ses tentacules dangereux (1999 : 64-65)
\end{abstract}

Déjà, une herbe émergeant verticalement figure une partie d'un monstre des profondeurs ; en 1944, dans Fragments d'un paradis, celui qui est devenu le traducteur de Moby Dick jouera encore à faire émerger les « lanières » d’un calamar géant, comme si la scène aquatique première, celle qui initie l'enfant à la jubilation créatrice, contenait en germe les motifs à venir. La puissance que le jeu procure au sujet lui reste en mémoire : «[...] je vois glisser sur l'eau calme du port, à la rencontre de leur destin, mes bateaux que le vent caresse. Tout le réel a disparu et je suis le dieu qui regarde ${ }^{6}(1999$ : 65). Du contact aquatique sont nés des créatures et un créateur. L'écoulement de l'eau suscite un devenir aléatoire, que

\footnotetext{
${ }^{1}$ Sonnet daté du 5 juillet 1911. Le biographe indique comme première publication sûre un autre sonnet, publié en mai 1913, évoquant un ruisseau. Il se réfère à H. Fluchère qui a fait connaître les poèmes dans le Bulletin $n^{\circ} 19$.

${ }^{2}$ Par exemple, à ses Stances (1899).

${ }^{3}$ Tant visuelles que métaphoriques.

${ }^{4}$ C’est une omniprésence, non une prédominance. Les « gionistes » ont montré l'importance extraordinaire de l'air.

${ }^{5}$ Giono en livrera deux versions ultérieures.

${ }^{6}$ C’est la fin du quatrième alinéa, intitulé « Poseidaon ».
} 
l'enfant suit avec passion et qu'il convertit en récit. La « naumachie » n'est pas ici un combat naval entre flottes adverses mais le périple aventureux de chaque bateau au fil de l'eau. Ce souvenir est la première exposition chez Giono du singulier procès d'émergence des images, qui est pour lui la création même, et qui s’opère lorsque la perception superpose deux plans, chacun supportant une image, l'une étant le spectacle que l'écrivain au travail a devant lui. Dès lors qu'une autre image lui apparaît, elle constitue un autre plan, celui des projections. Alors la fiction est enclenchée, des figures émergent et circulent. Or cet écran est aquatique. Giono le montre dans Noé, avec la frappante apparition du hussard, surgie alors que le narrateur voit la Méditerranée : « la mer toute nue montait si haut sur l’horizon qu'elle avait l'air d'être ainsi tenue lisse et verticale par une sorte de monstrueux mouvement giratoire [...] C’est là que je rencontrai pour la première fois ce personnage qui était comme un épi d’or sur un cheval noir. Il semblait être le fantôme des choses. » (1967 : 279). Ce grand pan de mer bleue ${ }^{7}$ apparaît au narrateur-promeneur alors qu’il contourne une vaste propriété nommée « Domaine Flotte », c'est dire combien la création romanesque est surdéterminée par l'eau... Dans le même ouvrage, Giono, pour traduire l'état de l'artiste au travail, recourt à l'élément, une eau interstitielle qui baigne le créateur absorbé :

Vous êtes assis à votre table du fond des eaux. Vous voyez toujours les quatre murs de votre chambre mais, entre les murs que vous regardez et vous, il y a de l'eau qui passe, s'installe, avec sa forme et sa couleur ; vous regardez par la fenêtre le paysage de votre village : les champs, les bosquets, les fermes autour, tout est englouti par de l'eau qui installe sur le visage familier des choses ses remous et ses mouvements (1961 : 22-23).

L’eau suscite la pulsion narrative et alimente le bain poïétique, le monde intérieur de l’artiste à l’œuvre.

La mention d’une eau donne souvent lieu à une dérive discursive ou au réamorçage du cours des événements, elle (re)lance le récit ${ }^{8}$. En cela aussi, elle est génétique. Dans Le Hussard sur le toit, c’est parce que Angelo entrant dans Manosque va à la fontaine qu'il est attrapé par une populace affolée par l'épidémie de choléra et persuadée de tenir un empoisonneur d'eau : « Il avait à peine plongé les mains dans l'eau du bassin qu’il se sentit brutalement saisi aux épaules, tiré en arrière, pendant que des bras forts le ceinturaient sans ménagement » (1989 : 254). Sauvé par un gendarme, au lieu de quitter la ville, Angelo, à nouveau pourchassé, gagne les toits. La fameuse séquence qui donne son nom au livre a pour origine le contact du héros avec l'eau de la fontaine. De même, dans Jean le Bleu et Le Chant du monde, la fontaine n’a rien d'une pièce décorative ${ }^{9}$; sa mention infléchit le cours du récit : lorsqu’un personnage y arrive, il est troublé par la sensation de renouer avec ce qui lui est essentiel. Dans Le Chant du Monde Antonio éprouve la double présence du fleuve et de Clara ; dans Jean le Bleu, l'anarchiste retrouve son exaltation pour la liberté (chapitre III) et dans une autre histoire enchâssée, les paroles du pêcheur-horloger Maillefer - un de ces travailleurs de l'eau dont Giono a le secret- relance une action qui était au point mort (chapitre VII).

L’eau est encore, elle-même, créatrice. Dans le récit autobiographique Jean le Bleu, le narrateur évoque la maison insalubre où il a grandi : «L’humidité montait dans les murs jusqu'au grenier » (1972 : 38). Sur l'un des murs le suintement dessine une figure. Cette paréidolie n’est pas une impression fugitive, elle devient « la dame du mur », fascine l'enfant qui se sent regardé en retour, éprouvant « l'émotion de son regard ». Un jour l'émotion culmine et c’est encore par l'eau : « Je regardais la dame. Il avait plu toute la nuit une sorte de pluie folle couchée par le vent montagnier et le mur du nord était humide. Une petite goutte d'eau perlait dans les deux yeux verts » (1972 :39). Le commerce de l'enfant avec la dame lui apprend que de l'eau naît la forme, le visage, qui n’est pas un simple contour plastique mais déjà un être ému et émouvant. A plusieurs reprises, parlant de lui en proie à l'émotion, Giono assimile son être intérieur à une eau. Ainsi, lorsqu'il se sent regardé par la « dame du mur » : « la pierre jetée dans cette flaque d’eau calme que j’étais c’était elle qui la jetait en me regardant » (1972 : 38). Il s’éprouve comme le site d'une vibration qui se propage. Cette sensation semble même le fin mot de son être, car Giono voit dans sa sensibilité exacerbée son principal trait de caractère. Il décrit : « cette sensualité qui faisait de moi une goutte d'eau traversée de soleil, traversée des formes et des couleurs du monde, portant, en vérité, comme la goutte d’eau, la forme, la couleur, le son, le sens marqué dans ma chair » (1972 : 96).

\footnotetext{
${ }^{7}$ Philippe Bonnefis, déjà, a calqué l'expression proustienne en intitulant son essai sur la couleur bleue (et la cyanose) Giono : Le petit pan de mer bleue.

${ }^{8} \mathrm{Il}$ arrive qu'à l'inverse la rencontre d'une eau donne lieu à une pause contemplative : le promeneur fait halte et se ressource littéralement en goûtant le compagnonnage de l'eau. On trouve de telles pauses (au bord d'un bassin, d'une fontaine) dans Noé ainsi que dans « Monologue » (Faust au village).

${ }^{9}$ Cela n’empêche pas l'écrivain, par ailleurs, d'évoquer la beauté de certaines fontaines, par exemple dans Colline ou dans « Monologue » (1978 : 23$)$.
} 


\section{Les eaux, des êtres vivants différenciés}

Giono différencie les eaux, les traite en êtres vivants dotés d’un tempérament, dont je présenterai quelques exemples, mais les détermine assez rarement par un hydronyme ${ }^{10}$. Dans sa notice relative au Chant $d u$ monde, Pierre Citron explique comment des réminiscences mêlées l'inspirent: « Ce fleuve innommé qui est l'axe du roman procède sans doute de la Durance, mais c'est une Durance grossie jusqu'à être une sorte de petit Rhône et sur laquelle jouent des souvenirs de Verdun, du Giffre qui coule près de Taninges, de l'Orbe, d'autres torrents sans doute et des évocations imaginaires des fleuves nordiques » (Citron in Giono, 1972 : 1271). Au même titre que les autres personnages, une eau sera plus ou moins saillante. Ici, notable, comme l'eau du Vaccarès, au delta du Rhône, sujet d'un grand portrait baroque mêlant règnes et éléments au point que son auteur commente les enchevêtrements: «Ce n’est plus $\mathrm{H}^{2} \mathrm{O}$, c’est Méduse » (1983:327). Ailleurs, insignifiante, comme dans ce croquis de L'Iris de Suze: "Des peupliers scintillants accompagnaient le serpentement d'un ruisseau sans histoire dans des prairies d'un vert acide » (1983: 518). Une des pluies de Que ma joie demeure se distingue par son irrégularité et son imprévisibilité: «C’était une pluie mince, pendue dans le ciel comme un serpent qui danse sur sa queue. Elle touchait trois plantes ici, trois plantes là, là un arbre, là le coin d’un champ. [...] » (1972 : 483). A l'opposé, la pluie diluvienne de Fragments d'un paradis est continue, pesante et monotone. Le fontainier dont l'écrivain recueille les paroles dans « L'eau vive » se souvient des facéties d'une fontaine rebelle :

Jamais plus de caprices que cette fontaine d'Observantines. Une chèvre, cette eau. J'allais, le matin, le long des rigoles ; [...] Vides, sèches. Je tournais le coin du cyprès : ma fontaine était là, ruisselante d'eau [...]. Eh bien, vous le croirez si vous voulez : [...] elle était venue débaucher en plein coteau un vieux gaillard de ru souterrain. Ils se mariaient là dans l'ombre. Et c'est bête, les ruisseaux de dessous terre ; il lui donnait toute son eau et elle s'en allait là-bas, près du cyprès, la gaspiller dans le soleil et dans le vent (1999 : 37-38).

Au début du film L'Eau vive, Giono parle en voix off de la Durance et du futur barrage de Serre-Ponçon: «Il n’est plus question de la laisser faire à sa tête. Ce caractère qui s'est exprimé librement pendant des milliers d'années, on veut le briser » (Villiers, 1958). L'écrivain avait déjà exposé sa conception dans le texte « Le chant du monde », qui clôt le recueil Solitude de la pitié, paru en 1932 : « Un fleuve est un personnage, avec ses rages et ses amours, sa force, son dieu hasard, ses maladies, sa faim d'aventures. Les rivières, les sources sont des personnages : elles aiment, elles trompent, elles mentent, elles trahissent, elles sont belles, elles s’habillent de joncs et de mousses » (1993 : 148-149). Ayant énuméré d'autres éléments naturels, il ajoute : «C'est une société d'êtres vivants » (1993 : 149).

La nature en effet forme un tout, composé mais symbiotique, au sein duquel tout communique. Chaque eau a sa flore et sa faune ${ }^{11}$, parfois particularisées à l'extrême. Dans Que ma joie demeure, l'écosystème de l'étang a son dialecte: « les loutres ne parlaient même pas ruisseau ou torrent, [...] elles parlaient étang Grémone » (1972 : 501). L’abondance de métaphores aquatiques, d'analogies réversibles, atteste les correspondances liant les êtres: tantôt un feuillage miroite comme une eau, tantôt une eau tamise la lumière comme un feuillage. La synergie de l'écosystème se manifeste dans la germination printanière (la dernière partie du Chant du monde) ou a contrario par le déchaînement des forces naturelles, qui suppose un dérèglement violent (l'orage final de Que ma joie demeure, la chute du glacier de Batailles dans la montagne). Dans Fragments d'un paradis le jeu du calamar géant avec les oiseaux de mer qui le picorent et le nettoient est d'abord décrit comme un système symbiotique ; il dégénère en énigme effrayante avec l'engluement des volatiles dans un liquide que le calamar sécrète abondamment.

Certaines eaux communiquent entre elles, l'une devient l'autre, la forme d'eau peut donc varier par échange. Giono décrit ainsi l'action d'une trombe orageuse comme le rapt d'une eau par une autre : "La nuée plongea sa tête dans le torrent et se mit à barboter et à boire comme une bête. Le lit de l'Ouvèze en resta à sec sur plus de vingt mètres, blanc comme un

\footnotetext{
${ }^{10}$ Giono baptise quelquefois ses eaux fictives (« Le Gaudissant » dans Regain) mais l'écrivain les nomme plus souvent dans ses chroniques et nouvelles (la fontaine des Observantins, le Vaccarès, le Rhône...) ; il lui arrive encore de désigner par un nom de propriétaire : « le bassin de Bouscarle » (« Monologue », Faust au village). Remarquons un fait unique dans les annales du récit maritime : si la destination du bateau est bien évoquée dans Fragment d’un paradis, on cherchera en vain le port de départ.

${ }^{11}$ Les différentes eaux trouvent à cet égard quelques traits typiques : les loutres et les carpes habitent l'étang, le congre, le fleuve, la mousse s'attache aux bassins et aux canons des fontaines, quant à la mer gionienne, elle abrite en particulier les monstres géants : raie et calamar des abysses.
} 
os pendant que tout gargouillait dans le nuage » (1972 :771-772). La trombe et la rivière sont ici à la fois des personnages différenciés et des formes de l'eau, vases communicants où passe la matière aquatique. De fait, en plus d'appartenir figure après figure à une « société d’êtres vivants », aux réseaux naturels, l’eau existe en tant que matière.

\section{Propriétés de l'eau gionienne}

Trois propriétés communes à toutes les eaux sont repérables, ce qui autorise à parler d'une matière aquatique : elle est bruyante, irisée, odorante.

L'eau se signale d'abord par son bruit. «C'était un murmure de roseaux et de claquements sur les pierres » lit-on à l'approche de l'étang Grémone (1972 : 483). Giono fait grand usage de la famille du mot «tambour », qui en devient emblématique, pour signifier la percussion fondamentale ${ }^{12}$, y compris celle de la mer. De singulières onomatopées marquent une présence aquatique vivante et puissante. Lorsque Bobbi, le héros de Que ma joie demeure, voit en rêve « le cœur de la rivière », il entend la pulsation du cœur battant : « plouf, plouf, plouf » (1972 : 619).

Après sa sonorité, l'eau se caractérise par sa teinte, rarement uniforme ; le moindre développement d'un narrateur s'attardant aux bords de l'eau le conduit à moduler les couleurs. Cette moire « accapare l'œil ». Bouslama, dans son étude des couleurs aquatiques, parle de la « force captante des eaux et leur capacité de mélange » (1984 : 59). La faune aquatique partage avec son élément la faculté de faire naître des coloris mobiles. Le chatoiement traduit le fin mouvement de l’être aquatique, une action en cours ou la vitalité latente. Il en résulte un aspect imprédictible qui fascine. Le phénomène est porté à son comble dans les visions marines de Fragment d'un paradis, qui vont jusqu'à offrir des couleurs « inconnues ». Le récit est en quasi-totalité composé des prémisses de l'émergence de monstres abyssaux. Au passage de la raie géante dans les eaux de surface « le bouillonnement de couleurs et d'éclairs illuminait si violemment la profondeur de la mer qu'il était possible de voir à des profondeurs considérables le balancement de longues algues [...] on vit par l'avant la mer toujours extraordinairement lisse se colorer, sur une très vaste étendue, de reflets de soie » (2002 : 29). Le romancier évoque des « ondes de couleurs » (2002 : 31) qui parcourent l'être inconnu, « une infinité de modulations transitoires » (Ibid.). Ainsi, il ne s'agit pas des couleurs de la bête, ni de celles de l'eau. Leur matière : l'eau et la chair des êtres de l'eau, est un espace traversé par les vibrations lumineuses. Même lorsque l'écrivain décrit la réflexion de l'eau, il laisse deviner une autre dimension de cette riche matière, qui absorbe et recèle davantage que son vis-à-vis. Quand la nuit tombe sur le delta du Rhône, le poète constate : « Les étoiles envahissent le delta. Il y a plus de constellations dans le Vaccarès que dans le ciel » $(1983: 330)$.

Vibrante de sons, parcourue de couleurs, l'eau est encore odorante. Lorsqu'elle n'est pas seulement une « odeur d'eau », la senteur est tantôt obscure et limoneuse, tantôt florale mais putride, en tout cas complexe, ce qui exclut la plupart du temps d'attacher à l'eau gionienne l'habituelle qualité de pureté. Dans plusieurs ouvrages, l'odeur de l'eau constitue une énigme ; l'investigation occupe longuement le narrateur, tel le narrateur proustien à la recherche de la source savoureuse. A l'issue de mille détours, le lecteur de Noé, celui de Fragment d'un paradis, apprendront enfin que la mystérieuse odeur, florale mais écœurante, est une odeur cadavérique ${ }^{13}$. Dans le récit maritime il faut attendre quarante pages pour que la première impression olfactive soit élucidée (2002 : 27 et 66). L’odeur diffusée par la créature marine est une odeur de chair en putréfaction. C’est une réminiscence de l'expérience des charniers de la grande guerre. Dans Noé, l'épisode horrifique de la nuit passée au domaine de la Thébaïde commence par la rencontre de l'eau ; le mystère de la « vilaine odeur », l’odeur « infernale » (1961 : 193) qui emplit la chambre qu'on lui donne, s'explique d'une manière atroce : une tache humide du matelas résultant de la liquéfaction partielle d’une morte, trop longtemps veillée dans le lit. Voilà donc le revers létal de la liquidité. Bien sûr, l'eau est à distinguer d'autres liquides. Mais l'épisode de la nuit à la Thébaïde est entièrement placé sous le signe de l'eau. Dans son souvenir le narrateur associe l'odeur au milieu aquatique. L'insistance sur le concert des batraciens et sur les bassins est éloquente: « la couleur de bronze de ces eaux plates, les mousses dont elles étaient chargées, la mélancolie dodelinante du chant des bêtes aquatiques : tout concourait à donner l'idée d'engloutissement. » (1961: 187-188).

\footnotetext{
${ }^{12}$ L'eau émet parfois une vibration. Cette vibration semble la part acoustique de l'ondoiement, dont la part visible sera le chatoiement. I. Bouslama voit l'ondulation comme un « mouvement primordial» $(1984: 13)$.

${ }^{13}$ Le narrateur de Noé suggère que l' « odeur si belle » qui passionnera Angelo dans le roman éponyme (laquelle n’est pas une odeur d'eau, mais le parfum de Pauline) serait la conversion de l’odeur de putréfaction qui le fit fuir la Thébaïde cette nuit-là.
} 
Il faut remarquer une dernière propriété de l'eau gionienne : l'intermittence. Continue et discontinue, l'eau possède virtuellement les deux facultés, qui s'expriment alternativement. Une eau peut disparaître sans cesser d'être. Dans le texte intitulé « Le temps », Giono reprend la traditionnelle comparaison en glissant au passage : « par la vitesse avec laquelle il passe, [le temps] donne [...] l'impression qu'il se perd, comme on dit d'une eau qu'elle se perd quand elle est bue par la terre (or c'est en fait la seule façon qu'elle a de ne pas se perdre) » (2006 : 70). Quand l'eau n'est plus, elle est encore. La plupart des commentateurs de Colline décrivent le fonctionnement en vases communicants de la fontaine et de Janet : la logorrhée du vieil homme se déclenche en effet quand tarit la fontaine des Bastides Blanches. Il faut dire que Giono conditionne cette lecture par tous les moyens, en premier lieu par les métaphores aquatiques. Alan J. Clayton, dans son essai « Pour une poétique de la parole chez Giono », considérant l’étroite homologie entre l'eau et la parole, relève: « la disparition de l'une appelle tout de suite le surgissement de l'autre » et constate : «l'eau souterraine dont aucun savoir humain n’arrivait à expliquer la disparition soudaine - car Lure est « la mère des eaux [...] se remet à couler sitôt que Janet se tait une fois pour toutes » (1978 : 42-43). Il faut pourtant préciser que dans la montagne de Lure, l'eau n’a pas cessé de couler.

Elle ne coule plus aux Bastides Blanches mais dans les ruines d'une autre fontaine. Gagou le simple, celui qui ne parle pas, mais bave, sait la trouver dans un village abandonné. Giono joue à brouiller les pistes de l'eau Cette quatrième propriété de la matière aquatique gionienne l'éloigne de la conception généralisante que s’en fait Bachelard lorsqu’il conduit son parallèle entre eau et parole : «L'eau est la maîtresse du langage fluide, du langage sans heurt, du langage continu, continué [...] qui donne une matière uniforme à des rythmes différents » (1996 : 209). Chez Giono, l'eau n’a rien d’uniforme. Sa faculté de disparition et de résurgence la définit au contraire et marque aussi bien les formes « eau de fontaine », « eau de source », « pluie », « rivière ». Elle peut tarir, couler goutte à goutte ou à torrent. Son débit varie d'un extrême à l'autre. Ceci est en premier lieu à mettre sur le compte d'une inspiration de la nature vécue. Sylvie Giono a parlé des habitudes de son père qui faisait presque tous les jours « [...] la promenade du canal d'arrosage qui passe juste au-dessus de la maison. C’est à flanc de colline [...] il y a une très belle vue sur la plaine de la Durance » (1995 : 19). Pour effacer la vieille étiquette « régionaliste », les critiques insistent toujours sur la dimension imaginaire de l'œuvre, son universalité, et sur la faculté de fabulation toute personnelle de Giono. En l’occurrence, son imagination aquatique est en rapport étroit avec l’expérience de la réalité géographique méridionale. La fréquentation de la nature provençale et alpestre, de ses habitants, qui pâtissent de la sécheresse, des crues, des violentes pluies automnales, la tradition orale, la présence des ouvrages d’irrigation, n’ont pas moins imprégné le Manosquin que le natif d’Aubagne Marcel Pagnol écrivant Manon des sources ${ }^{14}$, que André de Richaud rédigeant La Fontaine des lunatiques ou encore, évidemment, que Henri Bosco. Interrogé, en 1940, sur sa rencontre la plus émouvante, Giono évoque une promenade au cours de laquelle il est tombé sur « deux maisons séparées par un ruisseau, ou, plus exactement, la trace sèche d'un ruisseau. Il n’y coule que des abeilles sauvages et des lézards. Cependant, on entendait plus haut un bruit d’eau. Très léger [...] » L’écrivain ajoute : « J'en eus soif tout de suite. » (1982 : 9). S'ensuit l'histoire de la haine unissant deux habitants solitaires des maisons, l’un tenant la source au fusil pour empêcher l'autre d'y puiser. L’eau, trésor caché, est un topos méridional, voire méditerranéen, qui n’a rien d'exclusivement littéraire pour Giono : c’est une réalité sensible. L'extraordinaire vision du paysage de la chronique « Monologue » témoigne autant de son esprit d’observation que de sa capacité à extrapoler :

[...] Elle ne coule presque pas à la surface de la terre. L'herbe la garde dans son feutre et se la distille de tige à tige, de feuille à feuille, jusqu'aux racines qui la guident encore dans la terre pendant sept à huit centimètres, puis, à Dieu vat ! Elle s'enfonce toute seule mais sur des traces qui datent de qui sait combien de mille ans [...] il y a un hêtre formidable [...]. Le hêtre est si formidable parce qu'il peut boire à sa soif. [...]. Ses racines doivent descendre là-bas dedans aussi, en serpentant le long d'un pilier ou le long d'un mur, ou en plongeant carrément comme des câbles, depuis le plafond jusqu'à l'eau (1978: 21-22).

Ayant imaginé le vaste réservoir naturel qui expliquerait la vitalité végétale d’un pays dont la terre est si sèche, il développe encore : « [...] on peut presque se représenter les couloirs et les grandes salles dont je parle rien qu’en regardant ici dessus l'emplacement des massifs les plus touffus et les parcours des haies les plus noires. A ces endroits-là, on pourrait jouer sur le pays comme sur un tambour, le moindre coup retentirait dans des catacombes [...]» (1978 : 22-23). L'eau

${ }^{14}$ Film écrit et réalisé en 1952, avant d’être augmenté du tome Jean de Florette pour le dyptique romanesque que Pagnol intitulera L’Eau des collines. 
cachée est visible à qui sait lire son paysage. Giono représente les intermittences de l'eau, donne la parole à ceux qui la connaissent (le fontainier, l'aiguadier...) et en fait même la trame de son roman Colline.

\section{Références bibliographiques}

BACHELARD, Gaston (1996). L’Eau et les rêves. Paris : José Corti, coll. « Le Livre de poche ».

BELGHMI, Michèle (1987). Giono et la mer. Bordeaux : Presses Universitaires de Bordeaux.

Bouslama, Ibtissem (1984). La poétique de l'eau dans l'œuvre de Giono. Lectures de l'imaginaire. Thèse de doctorat. Université de Provence Aix-Marseille I.

Citron, Pierre (1990). Giono 1895-1970. Paris : Seuil.

Clayton, Alan J. (1978). Pour une poétique de la parole chez Giono. Paris : Lettres Modernes Minard.

Giono, Jean (1961). Noé, Paris : Gallimard, coll. « Le Livre de poche ».

- (1972). Euvres romanesque complètes II. Paris : Gallimard. Bibliothèque de la Pléiade. Contient : Jean le Bleu (éd. or. 1932), Le Chant du monde (éd. or. 1934), Que ma joie demeure (éd. or. 1934), Batailles dans la montagne (éd. or. 1937).

— (1978). Faust au village. Paris : Gallimard.

— (1982). « Une interview inédite (1940)». Dans : Bulletin 18, Association des Amis de Jean Giono, automne hiver 1982. 7-12.

— (1983). Euvres romanesque complètes VI. Paris : Gallimard, Bibliothèque de la Pléiade. Contient notamment L'Iris de Suze (éd. or. 1970).

— (1989). Le Hussard sur le toit. Paris : Gallimard, coll « Biblos ».

— (1993). Solitude de la pitié. Paris : Gallimard, coll. « Folio ».

— (1998). Colline. Paris : Grasset, coll. « Le Livre de poche ».

— (1999). Rondeur des jours. L'eau vive, I. Paris : Gallimard, coll. « L'Imaginaire ».

— (2002). Fragments d'un paradis. Paris : Gallimard, coll. « L’Imaginaire ».

— (2006). La Chasse au bonheur. Paris : Gallimard, coll. « Folio ».

— (2011). L'Homme qui plantait des arbres. Paris : Gallimard.

Giono, Sylvie (1995). « Portrait d’un père » dans Le Magazine littéraire, n³29. 18-22.

Plantier, René (1982). « La poétique du fleuve dans Le Chant du monde de Giono » dans Jean Giono. Bulletin, n¹7. 25-50.

VILLIERS, François (1958). L’Eau vive (film). Scénario et dialogues : Jean Giono. Adaptation d’Alain Allioux. 